\title{
A Cross-sectional Examination of the Association Between Parental Distress and the Well-being of Children With Neurodevelopmental Disorders During the COVID-19 Pandemic
}

Antonio Mendoza Diaz ( $\nabla$ a.mendozadiaz@unsw.edu.au )

UNSW Sydney

Lucy Tully

University of Sydney

Anne Masi

UNSW Sydney

Ping-I Lin

South Western Sydney Local Health District

Daryl Efron

Royal Children's Hospital

Sue Woolfenden

UNSW Sydney

Valsamma Eapen

UNSW Sydney

\section{Research Article}

Keywords: child mental health, child psychiatry, COVID-19, neurodevelopmental disorders, autism, ADHD, family systems, parental mental health, developmental disorders

Posted Date: September 27th, 2021

DOI: https://doi.org/10.21203/rs.3.rs-821990/v1

License: (c) (1) This work is licensed under a Creative Commons Attribution 4.0 International License.

Read Full License 


\section{Abstract}

Background: The COVID-19 pandemic and its concomitant impacts have been unevenly shared across society, this is particularly true for children with neurodevelopmental disorders (NDD), who often rely on a network of services and social connections for their well-being. Children with NDD during the COVID-19 pandemic have had worrying trends regarding their worsening well-being and poorer parental mental health. This study's aim was to test whether these two factors were associated in a survey of Australian parents.

Methods: This cross-sectional study of Australian parents of children with neurodevelopmental disorders ( $n=304$, ages $2-17,63.8 \%$ male) sought to test the association between parental distress (as captured by the Kessler- 6 distress scale) and two child outcomes, worsening NDD symptoms and worsening health behaviours (i.e., sleep, diet, exercise, and media use).

Results: Parent distress was significantly associated with a worsening of symptoms of NDD and associated mental health comorbidities, and poorer child well-being. These results were significant even when including other variables of interest such as the families' sociodemographic characteristics, indicating parental distress is a key factor for the well-being of children and parents.

Conclusions: Overall, this study found relatively high levels of parental psychological distress, anxiety and poor well-being and demonstrated that parental psychological distress was significantly associated with parent-reported worsening of child diagnostic symptoms as well as poorer child well-being in relation to sleep, diet, exercise, and use of electronics. Together these findings suggest that deteriorating parental mental health (distress) due to COVID-19 is associated with adverse child mental health symptoms and well-being, as indexed by the behaviours described above.

\section{Background}

The COVID-19 pandemic and its concomitant impacts have been unevenly shared across society, with priority populations and vulnerable groups bearing a disproportionate burden of the infections and the economic and societal consequences (1). Further, those with a disability have been reported to be impacted by a triple jeopardy of increased risk and poor outcomes from the infection, reduced access to health and rehabilitation care, and the negative social impact of the anti-contagion measures (2). In this regard, parents and caregivers of children with neurodevelopmental disorders (NDDs), such as autism and intellectual disability, may have been particularly impacted due to not being able to access the usual services to successfully manage their children's condition. Often this is compounded by the stress of home confinement and changes in routine with limited or no opportunities for accessing formal or informal supports. It is therefore likely that parents of children with an NDD may experience poor mental health and well-being during lockdown when compared to parents of typically developing children. Research to date has found that parents of children with NDDs report higher levels of stress (3) as well as increased severity of child symptoms during COVID-19 restrictions (4) compared to pre-pandemic levels. 
As parents of children with NDD have higher pre-pandemic levels of stress than parents of children without NDD $(5,6)$, these parents are more likely to experience a worsening of stress and mental health problems during the lockdown. Further, a study from China observed that parents of children with special needs had increased rates of mental health problems but this was particularly high for parents of children with certain conditions such as autism compared to parents of children with intellectual disability or hearing or vision impairment (7). While this suggests a unique relationship between the child's specific neurodevelopmental condition and parental mental health, the impact of parental mental health on the child's well-being is less clear. Thus, this paper sought to examine the mental health of parents of children with NDDs during the COVID-19 pandemic by examining the association between parental distress and child well-being.

Studies to date on children with NDD during the COVID-19 pandemic have found worrying trends regarding child well-being for children with NDDs and their parents. A cross-sectional survey of children with NDDs and neurotypical controls in the UK found that, while all children had worse emotional symptoms during the pandemic compared with pre-pandemic norms, those with NDDs had higher conduct problems and emotional symptoms and lower prosocial behaviours compared with neurotypical controls (3). Similarly, a study conducted in Italy found that many parents of children with autism reported more frequent and intense behaviour problems during the pandemic (8). Thus, adverse impacts of COVID-19 are not conscribed to children, and parents are also impacted.

Surveys of parents of children with NDDs have also found increased stress and poorer well-being during COVID-19. A survey in Italy found that both parents of children with and without NDD reported increased parental stress during the lockdown compared to before the pandemic. However, parental stress was higher in the NDD group, compared to the neurotypical group, both during and before the lockdown phase and heightened stress was predicted by worsening of child symptoms (9). These findings are consistent with a survey carried out in the UK which showed an increased level of mental health problems in caregivers of children and adults with an intellectual disability, relative to those without a disability (10). Differences were 2-3 times greater than reported in earlier pre-pandemic studies. A survey of parents of children with NDD in Italy also found moderate to high levels of clinical stress, anxiety and depression during the pandemic (11). Similarly, surveys of parents of children with autism conducted in Saudi Arabia $(12,13)$ and USA $(14,15)$ have found poorer parental mental health during the pandemic, especially for caregivers of younger children $(12,14)$ and parents of children with more severe symptoms $(14)$.

The distress and well-being of parents of children with NDD is important to study during the COVID-19 pandemic, given that increased parental stress and poorer mental health may have concomitant impacts on child well-being (16-18). Family systems models from the 1960 s and 1970 s clearly articulated the importance of the family unit - including the role of parents - in supporting the mental well-being of their children $(19,20)$. The association between parent's and children's mental health has continued to be the subject of study, and has consistently found that parental mental health plays an important role for children's well-being $(17,18)$. As an example, a recent study on the mental health problems of Syrian refugee children found that parental psychopathology was a significant predictor of children's mental 
health status, even after controlling for the children's own trauma experiences (21). The mechanisms through which parental distress may influence child mental health include via modelling of parental emotional reactions; changes in family dynamics, routines and relationships; and through changes in parenting (22), such as increased permissive, inconsistent or harsh parenting. For children with NDDs and their parents, who have more elevated mental health problems than the general population, parental distress in particular may represent an important target for interventions to improve child well-being.

The present study sought to expand on our initial survey of a sample of Australian families with children with NDD, which found almost two-thirds (64.5\%) of parents reported a worsening of their child's NDD symptoms and mental health co-morbidities due to the COVID-19 pandemic (4). There was also worsening across other indicators of child well-being, such as poorer diet, less exercise and sleep, and increased use of electronic devices such as television, computers and digital media. The aim of this study was to examine levels of parental psychological distress during the COVID-19 restrictions, and its association with parental sociodemographic characteristics and child factors, children's NDD symptoms and mental health comorbidities, as well as other indices of child well-being.

The specific objectives were to:

1. Examine the rates of parental psychological distress, poor well-being and anxiety

2. Examine whether parental psychological distress was associated with parental sociodemographic factors such as parental age, education, relationship status, and residential setting (urban vs. rural) and child factors (sex, age).

3. Examine whether parental psychological distress was associated with worsening of symptoms of child NDD or comorbid mental health conditions.

4. Examine whether parental psychological distress was associated with poor child well-being in the areas of sleep, diet, exercise and electronic media use.

These objectives informed three core hypotheses of the study. First, given previous research, it was expected that there will be high rates of parental psychological distress, anxiety and poor well-being (Hypothesis 1). Second, it was expected that parental psychological distress will show a significant positive association with a worsening of child NDD symptoms and mental health comorbidities (Hypothesis 2). Finally, we hypothesised that parental psychological distress will be associated with poorer sleep, diet, and exercise, and with greater media use in children (Hypothesis 3 ).

\section{Methods}

Australian parents and caregivers of children aged 2-17 years with a NDD were invited to complete a cross-sectional self-report survey as described in the study by Masi and colleagues (4). The online questionnaire included study-specific, fixed-response questions. The survey questions were piloted with clinicians, researchers, and a small group of parents $(n=20)$, with feedback resulting in adjustments to the survey. The survey was promoted through different service providers and support groups as described 
in our previous study (4). The survey was open for approximately 6 weeks between May and June 2020 . Survey questions included socio-demographic characteristics of children (age, sex, culturally and linguistically diverse background) and parents (relationship to child, single-parent household, employment, urban vs. rural location), along with questions and standardised instruments on child symptom severity and well-being, and parent well-being.

\section{Child symptom severity and well-being}

Caregivers were asked to report their child's NDD diagnosis/es (e.g., ADHD, Autism, Cerebral Palsy, intellectual disability, rare genetic condition, Tourette syndrome) along with any diagnosed comorbid mental health conditions (e.g., anxiety, obsessive compulsive disorder). A composite variable called 'number of diagnoses' was calculated, summing the number of diagnoses/conditions that a parent endorsed. For each NDD condition and comorbid mental health disorder, parents indicated how much their child's symptoms changed from before the pandemic to when the pandemic was at its worst on a 5point scale with 1 'symptoms much improved', 2 'symptoms somewhat improved', 3 'symptoms the same', 4 'symptoms somewhat worse', and 5 'symptoms much worse'. Responses of 'somewhat' or 'much worse' symptoms were combined as a single 'symptoms worsened' group. If parents endorsed any diagnostic/comorbid symptoms worsening, this was recoded to a single binary variable called 'child symptoms worsened' (any worsening yes/no).

Questions about child well-being asked the parents to rate the extent to which COVID-19 had led to: reductions in sleep quality; exercise decreased; a poorer diet; increased TV viewing or digital media use increased video-game use. These questions were responded to on a 5-point (1-5) Likert scale: 'strongly agree' to 'strongly disagree'. Responses to these questions were then summed to give a single scale for 'child well-being' (range 5-20, with lower scores indicating poorer well-being). The scale had a Cronbach's alpha for internal consistency of $a=0.648$.

\section{Parent well-being}

Caregivers reported on their current distress levels and well-being using three standardised instruments. Psychological distress was measured using the 6-item Kessler Psychological Distress Scale (23). This is a non-specific Likert scale covering: (1) feelings of nervousness, (2) hopelessness, (3) restlessness, (4) feeling that everything takes too much effort, (5) sadness, and (6) worthlessness. Scores greater than or equal to 14 were considered in the clinical range on this measure (24). Quality of Life was captured using the 5-item WHO Well-being Scale - WHO-5 (WHO-5, 1998), which ascertains subjective well-being in the past 2 weeks using 5 positively worded items on a scale from 5 (all of the time) to 0 (none of the time) for a total range of $0-25$, which was then multiplied by 4 to get a score out of 100. Items include: (1) I have felt cheerful and in good spirits, (2) I have felt calm and relaxed, (3) I have felt active and vigorous, (4) I woke up feeling fresh and refreshed, and (5) my daily life has been filled with things that interest me. Scores equal to or above 50 were considered in the low well-being range on this measure (25). Finally, Generalised Anxiety was captured with the 2-item General Anxiety Disorder Scale - GAD-2 (24). These items are: (1) Feeling nervous, anxious, or on edge, and (2) Not being able to stop or control worrying. 
GAD-2 total range from $0-6$, with scores $\geq 3$ suggesting anxiety symptoms are within the spectrum of clinical severity (24).

Additional questions were asked about whether parent's pre-existing mental health conditions had worsened due to COVID-19, whether pre-existing physical health conditions had worsened, whether changes due to COVID-19 had created financial problems for them or their families, and whether COVID19 had caused a decrease in caregiver supports and services. Questions were answered on a 5-point Likert scale, from ' 1 ' indicating Strongly Agree through to ' 5 ' indicating Strongly Disagree. These variables were also dichotomised for analyses, with Strongly Agree or Somewhat Agree categorised as indicative of COVID-19 impacts.

\section{Ethical approval}

All procedures performed in this study involving human participants were in accordance with the ethical standards of the institutional research committee and with the 1964 Helsinki declaration and its later amendments or comparable ethical standards. Ethical approval for this study was provided by the human research ethics committee at South West Sydney Local Health District (2020/ETH00975).

\section{Analysis plan}

Data were analysed using SPSS (v25). To examine the rates of parental psychological distress, anxiety and poor well-being (Objective 1), descriptive data analyses were conducted. To examine whether parental psychological distress (K6) was associated with parental socio-demographic (parent age, urban/rural settings, parent education and relationship status) and child factors (child sex and age), chisquare tests were conducted (Objective 2). To examine whether parent psychological distress was associated with the worsening of diagnostic/comorbid symptoms, a two-step logistic regression model was conducted (Objective 3), with the independent variables of parental psychological distress (K6), controlling for family socio-demographics and child factors (parent age, number of child diagnoses, child sex, single vs two parent family, metropolitan vs rural location, financial problems, decreased supports). These variables were selected as covariates given they have established relationships with children's mental health. The $\mathrm{K} 6$ was selected as the predictor variable as it indexed parental distress in the sample. Similarly, a linear regression predicting a worsening of child health-related behaviours (composite variable) was conducted to examine Objective 4.

\section{Results}

Family socio-demographic characteristics and child diagnoses for the 304 parents ( $94.7 \%$ female) who completed the survey and their children with NDD are shown in Table 1. Male children comprised two thirds of the cohort (66.9\%) and the average age was 9.7 years (range: $2-17$; standard deviation: 3.8). Most children were cared for by two caregivers (81.9\%). Overall, $43.5 \%$ of children had two or more siblings. There were almost equal number of participants from the metropolitan (50.7\%) and from 
towns/rural/regional areas of Australia. On average, children had 2.5 diagnoses for NDD or comorbid mental health conditions, with ADHD and Autism Spectrum Disorder the most common diagnoses.

Table 2 presents findings for parent and child mental health. More than half of the parents in the sample reported that their own mental health condition had worsened due to COVID-19 and just under half reported that their physical health condition had worsened. Overall, $43 \%$ indicated that COVID-19 had led to financial problems for their family. More than two-thirds (73\%) indicated that they had decreased caregiver supports and services as a result of COVID-19. In terms of the standardised measures of parental distress, almost half (47.7\%) had psychological distress scores (K6) above the clinical cut-off, and one-third (33.0\%) had a World Health Organisation Quality of Life Scale (WHO) above the clinical cutoff. Similarly, just over one-third had a cut off above the clinical level for generalised anxiety.

In terms of proportion of parents who reported worsening of child symptoms $62.8 \%$ of respondents endorsed items stating their child's symptoms were "somewhat worse" or "much "worse". A breakdown of these scores revealed $45.9 \%$ of parents reported reductions in sleep quality as a result of the COVID-19 pandemic, $70.1 \%$ reported reductions in their child's exercise, $33.2 \%$ reported a poorer diet, $82.6 \%$ reported children had been watching significantly more TV or digital media, and $65.1 \%$ reported significantly more video game use.

Chi-square tests were used to assess whether the distribution of parental psychological distress (K6) varied depending on a range of demographic variables: parent age, child sex, urban/rural location, education, and relationship status. Only parental age was significant $\left[\chi^{2}(5)=12.659, p=.027\right]$ with younger parents more likely to experience worse mental health outcomes. All other sociodemographic variables were non-significant, including child $\operatorname{sex}\left[\chi^{2}(3)=1.472, p=.689\right]$, urban/rural settings $\left[\chi^{2}(1)\right.$ $=.482, p=.488]$, parent's education $\left[\chi^{2}(4)=8.412, p=.078\right]$, and relationship status $\left[\chi^{2}(4)=6.412, p\right.$ $=.170]$. Since only parental age showed a significant association with parental distress the whole sample was included in subsequent analyses.

Table 3 shows the relationship between the continuous variables included in the analyses: child age, total number of diagnoses, parental psychological distress (K6), average worsening of symptoms, child wellbeing scale (i.e. sleep, diet, exercise, screen time and video game use), and detrimental effects associated with COVID: decreased caregiver supports and financial problems. This analysis was conducted to identify variables that needed to be controlled for in the regression model.

The correlation table above shows significant correlations among parental mental health and child outcomes. Parent psychological distress was significantly associated with agreement to statements indicating "decreased supports" and "greater financial problems". Parental distress was also associated with poorer child well-being (as indexed by the child health behaviours described above), and with parentreported worsening of children's symptoms.

The worsening of child symptoms (yes/no binary measure) and child well-being scale (continuous) were used as the dependent variables in a logistic regression (for symptom worsening) and a generalised 
linear regression respectively that included as dependent variables parental psychological distress (K6), child and parent age, parental education, total number of diagnoses, child sex, number of parents in the household, location (urban vs. rural), financial problems due to COVID, and loss of supports due to COVID. The results of these regressions are detailed in tables 4 and 5 .

As can be seen in Table 4, the logistic regression analysis showed that urban locations (as opposite to rural locations) were more strongly associated with a worsening of child symptoms. Further, parental psychological distress (K6) was a significant predictor of worsening of child symptoms. After controlling for the other variables in the analysis, the odds of reporting worsening child symptoms during COVID-19 increased 1.08 times for every increase in a point score on the parental psychological distress scale.

As shown in Table 5, the association between parental psychological distress and child well-being was replicated when predicting the impact of COVID-19 on child well-being (the scale comprising children's sleep, diet, exercise, and use of electronics). Parental psychological distress was significantly associated with poorer child well-being (decreased diet, sleep, exercise, and increased TV/digital media and video game use). No other variable significantly predicted a worsening of child well-being.

\section{Discussion}

This study aimed to describe the mental health of parents of children with neurodevelopmental disorders (NDDs) during the COVID-19 pandemic. It also aimed to examine whether increased parental psychological distress was associated with worsening of child NDD and comorbid mental health symptoms and child well-being. Overall, this study found relatively high levels of parental psychological distress, anxiety and poor well-being and demonstrated that parental psychological distress was significantly associated with parent-reported worsening of diagnostic symptoms as well as being significantly associated with poor child well-being in relation to sleep, diet, exercise, and use of electronics. Together these findings suggest that deteriorating parental mental health due to COVID-19 is associated with poorer child mental health and well-being.

More than half the sample parents reported clinical levels of psychological distress, and more than half reported that their mental health had worsened as a result of COVID-19 supporting Hypothesis 1.

Furthermore, more than one-third reported significant levels of anxiety and poor overall well-being. These findings are consistent with previous research suggesting that parents of children with autism and other neurodevelopmental conditions experience high levels of poor mental health a result of the COVID-19 pandemic (11-15).

This study also found that increased psychological distress was associated with a worsening of child NDD symptoms and other comorbid mental health conditions, as well as a poorer child well-being, supporting Hypothesis 2 and 3 . To the best of our knowledge, this is the first study to examine the association between parental distress and child mental health and well-being for children with NDDs in the context of the impact of COVID-19. Our findings suggest that poor parental mental health is associated with a worsening of child's neurodevelopmental symptoms during COVID-19 and poorer child 
well-being, such as decreased sleep and exercise, poorer diet and increased electronics use. It also suggests that parental mental health may be an important target for intervention to improve well-being of children with NDDs during COVID-19. In this study, parental psychological distress was significantly associated with decreased availability of services and supports, suggesting that service provision plays an important role in supporting vulnerable parents and families during the pandemic. In fact, as more than two-thirds of parents in this study (73\%) reported decreased supports and services during COVID-19, the need for continued and enhanced service provision is imperative. While services need to be universally available to families of children with NDD, there is also a need to target those who may be more vulnerable or at higher risk for poor outcomes. Previous research found that for children with autism, behaviour problems predating COVID-19 predicted a higher risk of more intense and frequent disruptive behaviour during the pandemic, suggesting those who were more vulnerable prior to the pandemic suffer worse outcomes (8).

These findings are aligned with family systems models, which posit that families undergo periods of transition as a unit $(20,26)$, and hence the experience of stressful situations impacts on the well-being of the whole family (19). Indeed, modern systems models had already predicted that the relationship between parents and their environment can ultimately impact the well-being of children (27). This is not to say that parental well-being is not important in its own right - it is; however, the current analysis focused on drawing attention to the fact that the impact of mental health distress experienced worldwide during the pandemic may ultimately be experienced most strongly by children. Indeed, this has been shown to be the case in studies of domestic violence during the pandemic, observing that often women and children are the victims of male violence $(28,29)$. These relationships exemplify the dynamic nature of family systems, whereby parental and child well-being is likely to interact as parents and children affect each other.

This study also explored whether parental well-being was associated with parents' own sociodemographic characteristics and child factors. Two findings warrant further discussion here. The first is the finding that younger parents tended to experience worse mental health outcomes. This could be due to the age of their children, with younger children demanding greater parental resources. In addition, it may be the case that younger parents require more formal and informal support from professional services as well as extended family and social networks that became inaccessible during the pandemic. Thus, younger parents may be a priority target for interventions to support parents of children with NDDs during COVID-19.

The second finding warranting discussion is that rural families reported less worsening of neurodevelopmental symptoms than urban families during the pandemic. This could be due to enhancements in service provision and access for rural families made available through telehealth before or during the COVID-19 lockdown. On the contrary, urban families that were used to accessing face-toface services might have had less access to health services as these closed for in-person contact. 
It is important to note that we assumed a specific causal direction when examining findings related to parental psychological distress and child well-being; however, the analyses undertaken do not preclude a relationship in the reverse direction, as noted above in discussing the dynamic nature of family systems. That is, it could well be the case that parents of children with greater needs, who were most affected during the pandemic, were in turn increasingly distressed by their children's worsening symptoms. In support of this, a study conducted in Saudi Arabia found that an increase in severity of child ASD symptoms due to COVID-19 had a negative impact on parents' emotional well-being (12). In reality, it is likely that the relationship between parent and child well-being is bidirectional with stressed parents and children having reciprocal effects on one another's mental health (30), and the restrictions due to COVID19 impacting the well-being of both children and parents.

A limitation of this study is that reports of parental well-being and child health behaviours were solely based on parental reports, and therefore susceptible to reporter-bias. That is, parent's perceptions of their own distress could influence their perception of their children's presentation $(3,31,32)$. Alternatively, the fact that parents were forced to spend more time at home may have confronted them with their children's behavioural difficulties, which were previously less evident when children attended educational or therapeutic centres. A second limitation is that the survey sample cannot be said to be representative of any one neurodevelopmental group. Indeed, Autism, Tourette syndrome, ADHD and other conditions were all covered within the mailing groups used (4). Yet while these heterogeneous conditions cannot be said to accurately represent any one population group, together they constitute an important perspective for the study of the impacts of the COVID-19 pandemic on parents of children who have additional neurodevelopmental needs. This is especially true as neurodevelopmental disorders are often sidelined in discussions related to the impacts of the pandemic.

The findings of the current study are timely and important. It is important for governments and service organisations to recognise and respond to the distress experienced by parents of children with NDD, and ensure that services are made available for supporting them. Given that these caregivers have higher baseline levels of stress than the general population of caregivers $(5,6)$ and since their deteriorating mental health during COVID-19 may adversely impact children's well-being, the provision of accessible services and supports during the pandemic is critical.

\section{Declarations}

Ethics approval and consent to participate: Ethical approval was obtained from the South West Sydney Local Health District research ethics committee, as noted in the methods section. Informed consent was obtained from all participants prior to their completion of the surveys.

Consent for publication: Not Applicable.

Availability of data and materials: The datasets used and/or analysed during the current study are available from the corresponding author on reasonable request. 
Competing interests: The authors declare that they have no competing interests

Funding: This study was not funded by a specific grant or funding body, but the support of the University of New South Wales, Murdoch Children's Research Institute, University of Sydney, and the South West Sydney Local Health District was important for the success of the study.

Authors' contributions: AMD and LT jointly wrote the manuscript; AMD and PL worked on the analysis and results; $A M$ organised data collection and ethics and all authors (AMD, LT, AM, PL, DE, SW, VE) contributed to the design of the study, the interpretation of findings, and the preparation of the final manuscript.

Acknowledgements: We would like to acknowledge all participating families and all families with children with neurodevelopmental disorders who continue to struggle through this global pandemic. Authors would also like to thank disability service providers and support groups: ADHD Support Australia, Autism Advisory and Support Service, Autism Awareness Australia, The Cerebral Palsy Alliance, Children and Young People with Disability Australia, Fragile X Association, Genetic and Rare Disease Network, Obsessive Compulsive and Related Disorders (OCRDs) Consortium, parents of children with tic disorders, parents of children with PANS/PANDAS, Raising Children's Network, Rare Voices Australia, Tourette Syndrome Association Australia. We would also like to acknowledge this study was conducted in the lands of the Aboriginal people of Australia, the traditional custodians of the land.

\section{References}

1. Wilder JM. The Disproportionate Impact of COVID-19 on Racial and Ethnic Minorities in the United States. Clinical Infectious Diseases. 2021;72(4):707-9.

2. Shakespeare T, Ndagire F, Seketi QE. Triple jeopardy: disabled people and the COVID-19 pandemic. The Lancet. 2021;397(10282):1331-3.

3. Nonweiler J, Rattray F, Baulcomb J, Happé F, Absoud M. Prevalence and Associated Factors of Emotional and Behavioural Difficulties during COVID-19 Pandemic in Children with Neurodevelopmental Disorders. Children. 2020;7(9).

4. Masi A, Mendoza Diaz A, Tully L, Azim SI, Woolfenden S, Efron D, et al. Impact of the COVID-19 pandemic on the well-being of children with neurodevelopmental disabilities and their parents. Journal of Paediatrics and Child Health. 2021;n/a(n/a).

5. Estes A, Olson E, Sullivan K, Greenson J, Winter J, Dawson G, et al. Parenting-related stress and psychological distress in mothers of toddlers with autism spectrum disorders. Brain and Development. 2013;35(2):133-8.

6. Hayes SA, Watson SL. The Impact of Parenting Stress: A Meta-analysis of Studies Comparing the Experience of Parenting Stress in Parents of Children With and Without Autism Spectrum Disorder. Journal of autism and developmental disorders. 2013;43(3):629-42. 
7. Chen SQ, Chen SD, Li XK, Ren J. Mental Health of Parents of Special Needs Children in China during the COVID-19 Pandemic. Int J Environ Res Public Health. 2020;17(24).

8. Colizzi M, Sironi E, Antonini F, Ciceri ML, Bovo C, Zoccante L. Psychosocial and Behavioral Impact of COVID-19 in Autism Spectrum Disorder: An Online Parent Survey. Brain Sciences. 2020;10(6).

9. Bentenuto A, Mazzoni N, Giannotti M, Venuti P, de Falco S. Psychological impact of Covid-19 pandemic in Italian families of children with neurodevelopmental disorders. Research in Developmental Disabilities. 2021;109:103840.

10. Willner P, Rose J, Stenfert Kroese B, Murphy GH, Langdon PE, Clifford C, et al. Effect of the COVID-19 pandemic on the mental health of carers of people with intellectual disabilities. Journal of Applied Research in Intellectual Disabilities. 2020;33(6):1523-33.

11. Grumi S, Provenzi L, Gardani A, Aramini V, Dargenio E, Naboni C, et al. Rehabilitation services lockdown during the COVID-19 emergency: the mental health response of caregivers of children with neurodevelopmental disabilities. Disability and Rehabilitation. 2021;43(1):27-32.

12. Alhuzimi T. Stress and emotional wellbeing of parents due to change in routine for children with Autism Spectrum Disorder (ASD) at home during COVID-19 pandemic in Saudi Arabia. Research in Developmental Disabilities. 2021;108:103822.

13. Althiabi Y. Attitude, anxiety and perceived mental health care needs among parents of children with Autism Spectrum Disorder (ASD) in Saudi Arabia during COVID-19 pandemic. Research in Developmental Disabilities. 2021;111:103873.

14. Manning J, Billian J, Matson J, Allen C, Soares N. Perceptions of Families of Individuals with Autism Spectrum Disorder during the COVID-19 Crisis. Journal of autism and developmental disorders. 2021;51(8):2920-8.

15. Kalb LG, Badillo-Goicoechea E, Holingue C, Riehm KE, Thrul J, Stuart EA, et al. Psychological distress among caregivers raising a child with autism spectrum disorder during the COVID-19 pandemic. Autism Research. 2021;n/a(n/a).

16. Leinonen JA, Solantaus TS, Punamäki R-L. Parental mental health and children's adjustment: the quality of marital interaction and parenting as mediating factors. Journal of Child Psychology and Psychiatry. 2003;44(2):227-41.

17. Solantaus T, Leinonen J, Punamaki R-L. Children's Mental Health in Times of Economic Recession: Replication and Extension of the Family Economic Stress Model in Finland. Developmental Psychology. 2004;40(3):412-29.

18. Fitzsimons E, Goodman A, Kelly E, Smith JP. Poverty dynamics and parental mental health: Determinants of childhood mental health in the UK. Social science \& medicine. 2017;175:43-51.

19. Bowen M. Family therapy in clinical practice. New York: Aronson; 1978.

20. Minuchin S. Families and family therapy. Massachusetts: Harvard University Press; 1974.

21. Eruyar S, Huemer J, Vostanis P. Review: How should child mental health services respond to the refugee crisis? 2018;23(4):303 - 12. 
22. Morris AS, Silk JS, Steinberg L, Myers SS, Robinson LR. The Role of the Family Context in the Development of Emotion Regulation. Social Development. 2007;16(2):361-88.

23. Kessler RC, Andrews G, Colpe LJ, Hiripi E, Mroczek DK, Normand S-L, et al. Short screening scales to monitor population prevalences and trends in non-specific psychological distress. 2002;32(6):95976.

24. Staples LG, Dear BF, Gandy M, Fogliati V, Fogliati R, Karin E, et al. Psychometric properties and clinical utility of brief measures of depression, anxiety, and general distress: The PHQ-2, GAD-2, and K-6. Gen Hosp Psychiatry. 2019;56:13-8.

25. Topp CW, Østergaard SD, Søndergaard S, Bech P. The WHO-5 Well-Being Index: A Systematic Review of the Literature. Psychotherapy and Psychosomatics. 2015;84(3):167-76.

26. Minuchin S, Baker L, Rosman BL, Liebman R, Milman L, Todd TC. A conceptual model of psychosomatic illness in children: Family organization and family therapy. Archives of General Psychiatry. 1975;32(8):1031-8.

27. Prime $\mathrm{H}$, Wade M, Browne DT. Risk and resilience in family well-being during the COVID-19 pandemic. American Psychologist. 2020;75(5):631-43.

28. Cappa C, Jijon I. COVID-19 and violence against children: A review of early studies. Child Abuse \& Neglect. 2021;116:105053.

29. Feder G, Lucas d'Oliveira AF, Rishal P, Johnson M. Domestic violence during the pandemic. BMJ. 2021;372:n722.

30. Patterson GR. Coercive family process. Eugene, OR: Castilia; 1982.

31. Briggs-Gowan MJ, Carter AS, Schwab-Stone M. Discrepancies among mother, child, and teacher reports: Examining the contributions of maternal depression and anxiety. Journal of abnormal child psychology. 1996;24(6):749-65.

32. Richters JE. Depressed mothers as informants about their children: A critical review of the evidence for distortion. Psychological bulletin. 1992;112(3):485-99.

\section{Tables}


Table 1

Sample socio-demographics and child diagnoses

\begin{tabular}{|c|c|c|c|}
\hline Variable & $\mathbf{N}$ & $\%$ & Mean (SD) \\
\hline Total sample size & 304 & & \\
\hline Parent Age & 304 & & \\
\hline Less than 20 & 8 & 2.6 & \\
\hline $20-29$ & 6 & 2.0 & \\
\hline 30-39 & 91 & 29.9 & \\
\hline $40-49$ & 161 & 53.0 & \\
\hline $50-59$ & 34 & 11.2 & \\
\hline 60 and above & 4 & 1.3 & \\
\hline Education & 304 & & \\
\hline Did not complete secondary school & 6 & 2.0 & \\
\hline Secondary school (Year 12) & 13 & 4.3 & \\
\hline Post-school certificate or diploma & 90 & 29.6 & \\
\hline Undergraduate Degree (University) & 108 & 35.5 & \\
\hline Postgraduate (e.g. Masters, PhD) & 87 & 28.6 & \\
\hline Relationship to child with NDD & 304 & & \\
\hline Mother & 284 & 93.4 & \\
\hline Father & 11 & 3.6 & \\
\hline Grandparent & 0 & 0.0 & \\
\hline Aunt/Uncle & 1 & 0.3 & \\
\hline Foster Parent & 4 & 1.3 & \\
\hline Other & 4 & 1.3 & \\
\hline Child Age & 301 & & $10.75(5.61)$ \\
\hline Child Sex & 304 & & \\
\hline Male & 194 & 63.8 & \\
\hline Female & 106 & 34.9 & \\
\hline Other & 3 & 1.0 & \\
\hline
\end{tabular}




\begin{tabular}{|llll|}
\hline Variable & N & $\%$ & Mean (SD) \\
\hline Prefer not to say & 1 & 0.3 \\
\hline Child Diagnoses & 304 & \\
\hline ADHD & 125 & 41.1 \\
\hline Anxiety & 103 & 33.9 \\
\hline ASD & 178 & 58.6 \\
\hline Cerebral Palsy & 34 & 11.2 \\
\hline Depression & 23 & 7.6 \\
\hline Genetic Disorders & 14 & 4.6 \\
\hline Intellectual Disability & 57 & 18.8 \\
\hline OCD & 33 & 10.9 \\
\hline Tourette's & 67 & 22.0 \\
\hline Other emotional or behavioural & 29 & 9.5 \\
\hline Rare genetic disorders & 23 & 7.6 \\
\hline Other diagnoses & 84 & 27.6 \\
\hline Total number of diagnoses & 304 & \\
\hline Num of parents in household & 304 & \\
\hline Single parent household & 60 & 19.7 \\
\hline Two-parent households & 244 & 80.3 \\
\hline Location & 304 & \\
\hline Urban (City or Town) & 153 & 50.3 \\
\hline Rural (Rural, Regional, or Remote) & 151 & 49.7 \\
\hline
\end{tabular}


Table 2

Questions assessing parent's and children's mental health

\begin{tabular}{|lccc|}
\hline Question & N & $\%$ & $\begin{array}{c}\text { Mean } \\
\text { (SD) }\end{array}$ \\
\hline Parental Questions & & & \\
\hline - My pre-existing mental health conditions have worsened due to COVID-19 & 212 & 58.0 & 3.33 \\
& & & $(1.97)$ \\
\hline - My pre-existing physical health conditions have worsened due to COVID- & 212 & 46.7 & 3.56 \\
\hline - Changes related to the COVID-19 crisis have created financial problems & 261 & 43.3 & 3.20 \\
for me or my family & & & $(1.61)$ \\
\hline - COVID-19 has caused decreased caregiver supports and services & 201 & 73.1 & 3.00 \\
\hline Child Questions & & & $(2.04)$ \\
\hline - Symptoms worsened (for any diagnosis) & 304 & 62.8 & \\
\hline Parental Well-being Scales & & & \\
\hline - K6 (Above cut-off) & 304 & 47.7 & 13.92 \\
\hline - WHO (Above cut-off) & & & $(5.17)$ \\
\hline - GAD-2 (Above cut-off) & 297 & 33.0 & 40.88 \\
\hline
\end{tabular}


Table 3

Correlation table of continuous variables

\begin{tabular}{|c|c|c|c|c|c|c|c|}
\hline & & 1 & 2 & 3 & 4 & 5 & 6 \\
\hline 1 & Child age & - & & & & & \\
\hline 2 & Total number of diagnoses & $.201^{\star \star}$ & - & & & & \\
\hline 3 & Decreased supports & .054 & -.098 & - & & & \\
\hline 4 & Fin problems & -.055 & -.095 & $.156^{\star}$ & - & & \\
\hline 5 & Parental distress (K6) & -.054 & -.013 & $-.248 * \star$ & $-.191^{\star \star}$ & - & \\
\hline 6 & Worse sym average & .017 & .103 & -.001 & -.076 & $.156^{\star \star}$ & - \\
\hline 7 & Worse child health beh & -.084 & $-.135^{\star}$ & $.180 \star \star$ & $.173^{\star \star}$ & $-.280 * *$ & $-.355^{\star \star}$ \\
\hline
\end{tabular}


Table 4

Logistic regression predicting the worsening of diagnostic symptoms

\begin{tabular}{|c|c|c|c|}
\hline & K6 & & \\
\hline Step $1^{\mathrm{a}}$ & OR $(95 \% \mathrm{Cl})$ & Df & Sig \\
\hline Parent Age & $1.806(.982,3.322)$ & 1 & .057 \\
\hline Number of diagnoses & $1.148(.951,1.387)$ & 1 & .151 \\
\hline Child sex (1 = Male; 2 = Female) & $1.254(.725,2.170)$ & 1 & .418 \\
\hline Number of parents ( 1 = Single; 2 = Two parent household) & $1.317(.648,2.677)$ & 1 & .446 \\
\hline Location (1 = Urban; 2 = Rural) & $.596(.353,1.006)$ & 1 & .053 \\
\hline Parental education & $.880(.657,1.177)$ & 1 & .389 \\
\hline Child age & $1.012(.955,1.071)$ & 1 & .690 \\
\hline Constant & 1.582 & 1 & .571 \\
\hline \multicolumn{4}{|l|}{ Step $2^{b}$} \\
\hline Parent Age & $1.587(.846,2.975)$ & 1 & .150 \\
\hline Sum of diagnoses & $1.176(.968,1.427)$ & 1 & .102 \\
\hline Child sex (1 = Male; 2 = Female) & $1.374(.783,2.410)$ & 1 & .268 \\
\hline Number of parents (1 = Single; 2 = Two parent household) & $1.280(.621,2.642)$ & 1 & .503 \\
\hline Location (1 = Urban; 2 = Rural) & $.538(.313, .925)$ & 1 & $.025^{\star}$ \\
\hline Parental education & $.986(.726,1.339)$ & 1 & .929 \\
\hline Child age & $1.013(.955,1.074)$ & 1 & .667 \\
\hline Financial problems $(0=\mathrm{No} ; 1=\text { Yes })^{\mathbb{}}$ & $.911(.766,1.083)$ & 1 & .291 \\
\hline Decreased supports $(0=\text { No; } 1=Y e s)^{\bigotimes}$ & $1.041(.908,1.194)$ & 1 & .564 \\
\hline Parental MH (K6) & $1.075(1.014,1.141)$ & 1 & $.016^{*}$ \\
\hline Constant & .439 & 1 & .433 \\
\hline \multicolumn{4}{|c|}{ 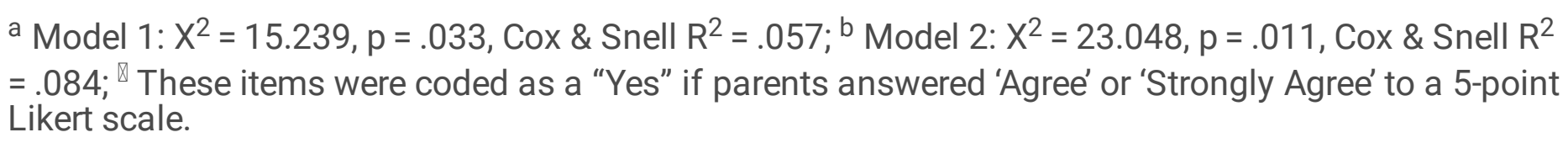 } \\
\hline
\end{tabular}


Table 5

Linear regression predicting the worsening of child health behaviours

\begin{tabular}{|c|c|c|c|c|}
\hline Variable & B (95\% Cl)* & Std. Error & Df & Sig \\
\hline Parent age & $.344(-.872,1.561)$ & .621 & 1 & .579 \\
\hline Parent education & $.224(-.382, .831)$ & .309 & 1 & .468 \\
\hline Sum of diagnoses & $-.275(-.648, .099)$ & .191 & 1 & .149 \\
\hline Child sex & $-.039(-1.156,1.078)$ & .570 & 1 & .945 \\
\hline Child age & $-.044(-.158, .071)$ & .058 & 1 & .453 \\
\hline Num of parents & $-1.052(-2.430, .325)$ & .703 & 1 & .134 \\
\hline Location & $-.012(-1.079,1.055)$ & .544 & 1 & .983 \\
\hline Fin problems & $.281(-.061, .623)$ & .175 & 1 & .107 \\
\hline Decreased supports & $.217(-.058, .492)$ & .140 & 1 & .122 \\
\hline Parental distress (K6) & $-.205(-.315,-.095)$ & .056 & 1 & $<.001$ \\
\hline Constant & $13.513(9.451,17.575)$ & 2.072 & 1 & $<.001$ \\
\hline
\end{tabular}

